This item was submitted to Loughborough's Research Repository by the author.

Items in Figshare are protected by copyright, with all rights reserved, unless otherwise indicated.

\title{
A joint coded two-step interference cancellation technique for a multiuser MIMO-SC-FDE system.
}

PLEASE CITE THE PUBLISHED VERSION

PUBLISHER

(c) IEEE

VERSION

VoR (Version of Record)

\section{LICENCE}

CC BY-NC-ND 4.0

\section{REPOSITORY RECORD}

Aljohani, Abdullah, and Jonathon Chambers. 2019. "A Joint Coded Two-step Interference Cancellation Technique for a Multiuser MIMO-SC-FDE System.”. figshare. https://hdl.handle.net/2134/5592. 
This item was submitted to Loughborough's Institutional Repository (https://dspace.lboro.ac.uk/) by the author and is made available under the following Creative Commons Licence conditions.

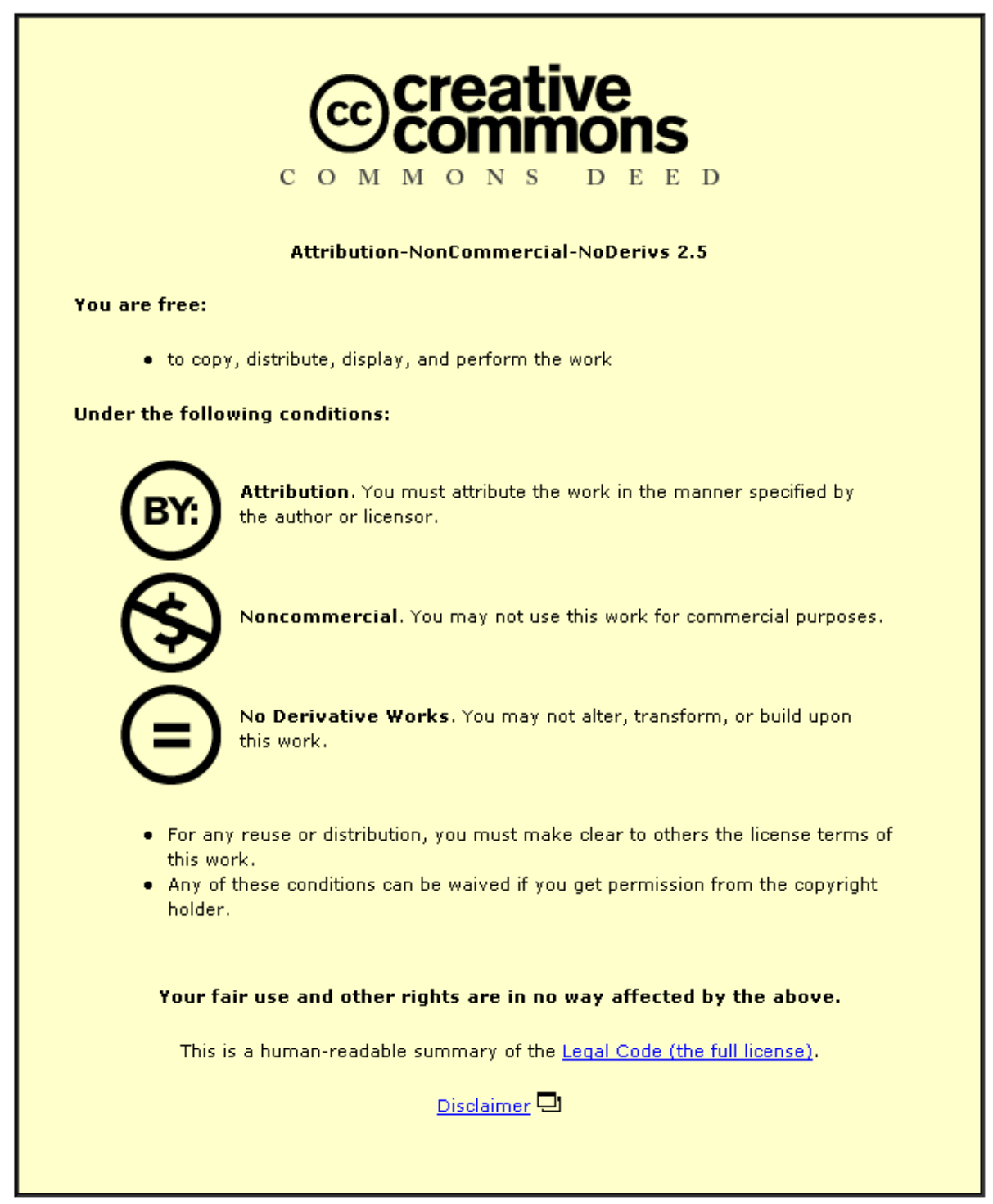

For the full text of this licence, please go to: http://creativecommons.org/licenses/by-nc-nd/2.5/ 


\title{
A Joint Coded Two-Step Interference Cancellation Technique for A Multiuser MIMO-SC-FDE System
}

\author{
A.Aljohani and J.Chambers \\ Advanced Signal Processing Group \\ Department of Electronic and Electrical Engineering \\ Loughborough University \\ Leicestershire, LE11 3TU \\ email:\{a.aljohani,j.a.chambers\}@lboro.ac.uk
}

\begin{abstract}
In this paper, we present a transceiver design for a broadband multiuser-MIMO communication system, where the co-channel users are equipped with multiple transmit and multiple receive antennas. In particular, we propose a joint coded two-step interference cancellation scheme which effectively combats the effect of co-channel interference (CCI) and inter-symbol interference (ISI) in multipath channels. To overcome the peak-to-average-power ratio limitation of multicarrier systems, the scheme employs single-carrier frequency-domain equalization (SC-FDE) based modulation combined with time-reversed space-time block codes (TR-STBC). The receiver performs minimum mean-squared error (MMSE) interference suppression at the first stage, and then, CCI cancellation is implemented with a bank of single-user channel decoders. Also, forward error correction outer convolutional encoding, and random bit interleaving are used to correct the carrier in deep fades. To verify the utility of the proposed approach, the paper also includes two sets of average symbol error rate simulations for two different channel models. The first set is for a frequency selective quasi-static fading channel while the second set is for a Rayleigh fading channel modeled by a modified Jakes' model, both of which confirm the advantage of the proposed scheme.
\end{abstract}

\section{Introduction}

Wireless communication systems are envisioned to offer broadband multimedia services such as mobile computing and high-speed wireless internet access to a large population of mobile users. This trend is driving communication systems towards higher data rate and enhanced quality, thus raising the difficulty to ensure reliable communi- cations over the shared bandwidth. A fundamental challenge for high data-rate transmission is inter-symbol interference (ISI) due to multipath dispersion. Single-carrier frequency-domain equalization (SC-FDE)-based multipleinput-multiple-output (MIMO) systems have been shown to be an effective solution with relatively low complexity in combating ISI whilst exploiting multi-antenna diversity gain. Moreover, MIMO-SC-FDE systems are an attractive solution for broadband wireless communications in particular due to their ability to overcome the peak-toaverage-power ratio limitation in multicarrier systems such as those based on orthogonal frequency-division multiplexing (OFDM) [1]. On the other hand, the SC-FDE-based time-reversal space time block coding (TR-STBC) scheme [2][3] is a potential candidate for use within broadband wireless access systems, such as IEEE 802.16 [4] [5]. The use of MIMO-SC-FDE with TR-STBC coding, transmits the data blocks from different transmit antennas. The orthogonality in the frequency domain implies that the effects of the channel at different frequencies are decoupled and easily equalized, but additional processing is required to remove CCI.

The many advantages of using MIMO-SC-FDE for a single user can also be extended to multiuser scenarios, where the sum capacity grows linearly with the minimum of $K$ and $n_{t}$ ( $K$ is the number of users communicating with a base station using $n_{t}$ transmit antennas) [6].

A minimum mean-square error (MMSE) interference suppression technique is developed in [7] where the interference from $K-1$ co-channels could be perfectly suppressed while providing a diversity order of $n_{t} \times\left(m_{r}-K+\right.$ $1)$ where $m_{r}(\geq K)$ is the number of receive antennas. In addition, a two-step iterative interference cancellation (IC) and ML decoding algorithm that provides additional performance gain over the basic MMSE scheme was given in [8] for a MIMO-OFDM system. To the best of our knowl- 


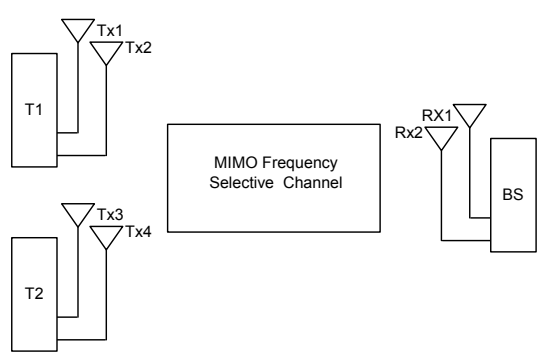

\section{Figure 1. The general layout of the multiuser system, two terminals and a base station, each equipped with two antennas.}

edge, no previous work has been reported in the literature on combining MIMO-TR-STBC, SC-FDE-MMSE and the two-step cancelation method to realize the combined benefits of these schemes, which is the objective of this paper.

In this paper, therefore, a joint MMSE interference cancellation scheme is considered for a broadband uplink system with $K$ synchronous co-channel users, each is equipped with time reversed space-time block coded (TR-STBC) transmit antennas. It is assumed that the frequency response of the channel remains constant over two consecutive blocks, i.e. a quasi-static channel, and we propose to employ TR-STBC over two consecutive time-domain blocks. The number of transmit antennas per user is limited in this paper to $n_{t}=2$, but the model can be generalized. In this proposed scheme we build upon the narrow-band uncoded interference suppression and cancellation scheme proposed in [7] to suppress the co-channel interference and inter-symbol interference simultaneously.

The remainder of this paper is organized as follows. We start in Section II by describing our system model. In Section III we propose the two-step interference cancellation. Simulation results are presented in Section IV and the paper is concluded in Section V.

Notation: Bold upper case $\mathbf{X}$ denotes a matrix or a frequency domain vector and lowercase $\mathbf{x}$ denotes a column vector. $\mathbf{X}^{j}$ and $\mathbf{x}^{j}$ denote the signal matrix and vector corresponding to the $j$ th user. We use $x_{k}$ to denote the $k$ th element of the time domain vector $\mathbf{x}$ of size $N$, where $k=0,1, \ldots, N-1$ and similarly an upper case letter to denote elements of frequency domain vectors. $\mathbf{x}_{n}$ denotes the $n$th block vector in the data stream. The matrix indexed by $p$ and $q$ is denoted by $\mathbf{X}_{p q}$. $\mathbf{I}_{N}$ is an identity matrix of size $N$. Complex conjugation, transposition and conjugate transposition of a matrix are respectively denoted by $(.)^{*}$, $(.)^{T}$ and $(.)^{H} . E\{$.$\} and \|.\|^{2}$ denote respectively the statistical expectation operator and squared Euclidean norm operator.

\section{SYSTEM MODEL}

We consider a 2-user space-time block coded SC-FDE wireless communication system, where each user terminal is equipped with $n_{t}=2$ transmit antennas. First, the incoming data stream is encoded by a half rate and constraint length of three convolutional encoder [9]. A random bit interleaver is then applied to the convolutional encoder output which is mapped as BPSK (here, BPSK is used for illustration, extension to other constellations is straightforward). Then, space-time block encoding processing, discussed further below, is implemented [3].

The $j$-th user's input is assumed to be divided into consecutive length $N$ blocks $\mathbf{x}_{(2 i)}^{j}$ and $\mathbf{x}_{(2 i+1)}^{j}$ where $i=$ $0,1,2, \cdots$. Each such pair of blocks forms the input to the block TR-STBC encoder whose output across the two antennas is given by

$$
\left[\begin{array}{ll}
\mathbf{s}_{2 i}^{j}(1) & \mathbf{s}_{2 i+1}^{j}(1) \\
\mathbf{s}_{2 i}^{j}(2) & \mathbf{s}_{2 i+1}^{j}(2)
\end{array}\right]:=\left[\begin{array}{cc}
\mathbf{x}_{2 i}^{j} & -\mathbf{P}\left(\mathbf{x}_{2 i+1}^{j}\right)^{*} \\
\mathbf{x}_{2 i+1}^{j} & \mathbf{P}\left(\mathbf{x}_{2 i}^{j}\right)^{*}
\end{array}\right]
$$

where $\mathbf{P}$ performs the modulo $N$ time reversal of a vector $\mathbf{x}$, i.e. $\mathbf{P x}=\left[\begin{array}{lllll}x_{0} & x_{N-1} & x_{N-2} \cdots & x_{2} & x_{1}\end{array}\right]^{T}$, and the two rows correspond to the first and second antennas. Before transmission, length $C P$ cyclic prefixes are added to each of the length $N$ coded blocks on the LHS of eq.(1) to avoid inter-block interference (IBI).

At the receiver, these cyclic prefixes are removed prior to calculating DFTs to perform frequency domain equalization. We assume that the frequency domain representations of the frequency selective channels between the two transmit antennas denoted, $g$, of the $j$-th user and the two antennas of the receiver, denoted $k$, are given by $H_{k g}\left(\omega_{l}\right)$ where $k, g=1,2$ and $\omega_{l}$ is the discrete frequency index with $l=0,1, \cdots, N-1$. These channels are assumed fixed for pairs of blocks $2 i$ and $2 i+1$. We assume DFTs of the vector elements of eq.(1) are given by

$$
\left[\begin{array}{ll}
\mathbf{S}_{2 i}^{j}(1) & \mathbf{S}_{2 i+1}^{j}(1) \\
\mathbf{S}_{2 i}^{j}(2) & \mathbf{S}_{2 i+1}^{j}(2)
\end{array}\right]=\left[\begin{array}{ll}
\mathbf{F s}_{2 i}^{j}(1) & \mathbf{F s}_{2 i+1}^{j}(1) \\
\mathbf{F s}_{2 i}^{j}(2) & \mathbf{F s}_{2 i+1}^{j}(2)
\end{array}\right]
$$

where $\mathbf{S}_{2 i}^{j}(1)=\left[S_{2 i}^{j}\left(1, \omega_{0}\right), S_{2 i}^{j}\left(1, \omega_{1}\right), \cdots, S_{2 i}^{j}\left(1, \omega_{N-1}\right)\right]^{T}$, $\mathbf{S}_{2 i}^{j}(2)=\left[S_{2 i}^{j}\left(2, \omega_{0}\right), S_{2 i}^{j}\left(2, \omega_{1}\right), \cdots, S_{2 i}^{j}\left(2, \omega_{N-1}\right)\right]^{T}$ and F denotes the $N \times N$ DFT matrix. Due to the time domain permutations and conjugate operations in the RHS of eq.(1), $\mathbf{S}_{2 i+1}^{j}(1)=-\left(\mathbf{S}_{2 i}^{j}(2)\right)^{*}$ and $\mathbf{S}_{2 i+1}^{j}(2)=\left(\mathbf{S}_{2 i}^{j}(1)\right)^{*}$. Thus, a block Alamouti matrix is formed.

Due to the orthogonality of the DFT the received frequency domain signals can be considered independently at each discrete frequency, so that for the first receive antenna at time block $2 i$ the received complex scalar frequency do- 
main value is given as

$$
\begin{aligned}
R_{2 i}\left(1, \omega_{l}\right) & =H_{11}\left(\omega_{l}\right) S_{2 i}^{j}\left(1, \omega_{l}\right)+H_{12}\left(\omega_{l}\right) S_{2 i}^{j}\left(2, \omega_{l}\right) \\
& +G_{11}\left(\omega_{l}\right) S_{2 i}^{j^{\prime}}\left(1, \omega_{l}\right)+G_{12}\left(\omega_{l}\right) S_{2 i}^{j^{\prime}}\left(2, \omega_{l}\right) \\
& +V_{2 i}\left(1, \omega_{l}\right) \forall \omega_{l}
\end{aligned}
$$

where $G_{11}\left(\omega_{l}\right)$ and $G_{12}\left(\omega_{l}\right)$ are equivalent channel coefficients for the second user and $V_{2 i}\left(\omega_{l}\right)$ is a complex noise term. Similarly, at time block $2 i+1$ as

$$
\begin{aligned}
R_{2 i+1}\left(1, \omega_{l}\right) & =-H_{11}\left(\omega_{l}\right)\left(S_{2 i}^{j}\left(2, \omega_{l}\right)\right)^{*} \\
& +H_{12}\left(\omega_{l}\right)\left(S_{2 i}^{j}\left(1, \omega_{l}\right)\right)^{*} \\
& -G_{11}\left(\omega_{l}\right)\left(S_{2 i}^{j^{\prime}}\left(2, \omega_{l}\right)\right)^{*} \\
& +G_{12}\left(\omega_{l}\right)\left(S_{2 i}^{j^{\prime}}\left(1, \omega_{l}\right)\right)^{*} \\
& +V_{2 i+1}\left(1, \omega_{l}\right) \forall \omega_{l}
\end{aligned}
$$

where $j$ and $j^{\prime}$ denotes respectively user 1 and 2 .

Equations (3) and (4) can be conveniently written in a matrix form as

$\mathbf{R}\left(1, \omega_{l}\right)=\mathbf{H}_{1}\left(\omega_{l}\right) \mathbf{S}^{j}\left(\omega_{l}\right)+\mathbf{G}_{1}\left(\omega_{l}\right) \mathbf{S}^{j^{\prime}}\left(\omega_{l}\right)+\mathbf{V}_{1}\left(\omega_{l}\right) \forall \omega_{l}$

where $\mathbf{R}\left(1, \omega_{l}\right)=\left[R_{2 i}\left(1, \omega_{l}\right), R_{2 i+1}^{*}\left(1, \omega_{l}\right)\right]^{T}$, is the received signal at the first receive antenna for the particular frequency, $\mathbf{S}^{j}\left(\omega_{l}\right)=\left[S_{2 i}^{j}\left(1, \omega_{l}\right), S_{2 i}^{j}\left(2, \omega_{l}\right)\right]^{T}$, is the signal from the first user $\mathbf{S}^{j^{\prime}}\left(\omega_{l}\right)=\left[S_{2 i}^{j^{\prime}}\left(1, \omega_{l}\right), S_{2 i}^{j^{\prime}}\left(2, \omega_{l}\right)\right]^{T}$, is the signal from the second user $\mathbf{V}_{1}\left(\omega_{l}\right)=$ $\left[V_{2 i}\left(1, \omega_{l}\right), V_{2 i+1}^{*}\left(1, \omega_{l}\right)\right]^{T}, \quad$ is the noise vector, $\mathbf{H}_{1}\left(\omega_{l}\right)=\left[\begin{array}{cc}H_{11}\left(\omega_{l}\right) & H_{12}\left(\omega_{l}\right) \\ H_{12}^{*}\left(\omega_{l}\right) & -H_{11}^{*}\left(\omega_{l}\right)\end{array}\right]$, is the first user channel and $\mathbf{G}_{1}\left(\omega_{l}\right)=\left[\begin{array}{cc}G_{11}\left(\omega_{l}\right) & G_{12}\left(\omega_{l}\right) \\ G_{12}^{*}\left(\omega_{l}\right) & -G_{11}^{*}\left(\omega_{l}\right)\end{array}\right]$ is the second user channel.

Similarly for the second receive antenna at the time blocks $2 i$ and $2 i+1$, a matrix equation becomes

$\mathbf{R}\left(2, \omega_{l}\right)=\mathbf{H}_{2}\left(\omega_{l}\right) \mathbf{S}^{j}\left(\omega_{l}\right)+\mathbf{G}_{2}\left(\omega_{l}\right) \mathbf{S}^{j^{\prime}}\left(\omega_{l}\right)+\mathbf{V}_{2}\left(\omega_{l}\right) \forall \omega_{l}$

Then the overall received frequency domain signal $\mathbf{R}\left(\omega_{l}\right)=\left[\mathbf{R}\left(1, \omega_{l}\right)^{T}, \mathbf{R}\left(2, \omega_{l}\right)^{T}\right]^{T}$ can be obtained as

$$
\begin{aligned}
\mathbf{R}\left(\omega_{l}\right) & =\left[\begin{array}{ll}
\mathbf{H}_{1}\left(\omega_{l}\right) & \mathbf{G}_{1}\left(\omega_{l}\right) \\
\mathbf{H}_{2}\left(\omega_{l}\right) & \mathbf{G}_{2}\left(\omega_{l}\right)
\end{array}\right]\left[\begin{array}{c}
\mathbf{S}^{j}\left(\omega_{l}\right) \\
\mathbf{S}^{j^{\prime}}\left(\omega_{l}\right)
\end{array}\right]+\left[\begin{array}{l}
\mathbf{V}_{1}\left(\omega_{l}\right) \\
\mathbf{V}_{2}\left(\omega_{l}\right)
\end{array}\right] \\
& =\mathbf{H}\left(\omega_{l}\right) \widetilde{\mathbf{S}}\left(\omega_{l}\right)+\mathbf{V}\left(\omega_{l}\right) \forall \omega_{l}
\end{aligned}
$$

and the columns of $\mathbf{H}\left(\omega_{l}\right)$ are denoted as $\overline{\mathbf{H}}_{i}\left(\omega_{l}\right), i=$ $1,2,3,4$.

We stress that in the context of an SC-FDE scheme when we decode $\mathbf{R}\left(\omega_{l}\right)$ in eq.(7), frequency-by-frequency, a following inverse DFT (IDFT) stage is required to return to the time-domain signal symbol. This is accounted for in the following section.

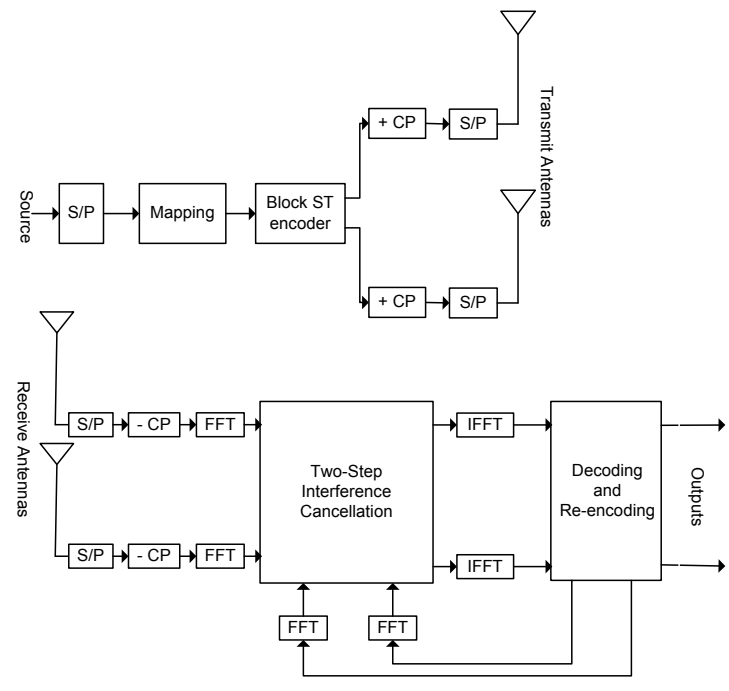

Figure 2. The MIMO SC-FDE System Model.

\section{TWO STEP INT. CANCELLATION}

We extend the two-step generalized MMSE interference receiver in [7] to the proposed two user TR-STBC-SCFDE scheme. For simplicity, we again consider the twouser scenario. The receiver structure is illustrated in Fig 2, where the DFT and IDFT operations are represented as Fast Fourier transforms (FFT).

In this receiver structure, the received signal will be passed through the following two parts of processing:

1. Soft signal value estimation based on a combined MMSE and multiuser detector followed by Viterbi decoder in the time domain.

2. Multiple-access interference cancellation and symbol re-estimation using the modified interference-free received signal.

\subsection{Combined MMSE and Multiuser De- tector}

Consider the $j$ th user terminal, where $j=1,2$, and define the following cost function to minimize the mean squared error caused by co-channel interference and noise in the symbol estimate $\hat{\mathbf{S}}^{j}\left(\omega_{l}\right)$, where $\hat{\mathbf{S}}^{j}\left(\omega_{l}\right)=$ $\mathbf{W}\left(\omega_{l}\right) \mathbf{R}\left(\omega_{l}\right)$ is the estimation of the frequency domain symbol $\mathbf{S}^{j}\left(\omega_{l}\right): J\left(\mathbf{W}\left(\omega_{l}\right)\right)=E\left\{\| \mathbf{S}^{j}\left(\omega_{l}\right)-\right.$ $\left.\mathbf{W}\left(\omega_{l}\right) \mathbf{R}\left(\omega_{l}\right) \|^{2}\right\} \quad \forall \quad \omega_{l}$. To minimize the mean squared error, we choose the weight matrix $\mathbf{W}\left(\omega_{\mathbf{l}}\right)$ of size $2 \times 4$ based on standard minimization: $\frac{\partial J\left(\mathbf{W}\left(\omega_{l}\right)\right)}{\partial \mathbf{W}\left(\omega_{l}\right)^{H}}=\mathbf{0}$.

From eq. (7) consider $\mathbf{M}\left(\omega_{l}\right)=\mathbf{H}\left(\omega_{l}\right) \mathbf{H}^{H}\left(\omega_{l}\right)+\frac{1}{\tau} \mathbf{I}_{\mathbf{2 m}}$ where $\tau=E_{s} / N_{0}$ is signal to noise ratio at the particular 
frequency $\left(\omega_{l}\right)$, and the structure of the TR-STBC implies the block-diagonal elements of $\mathbf{H}\left(\omega_{l}\right) \mathbf{H}^{H}\left(\omega_{l}\right)$ are diagonal, the block off-diagonal elements could be approximated by zero values but complexity reduction is not the focus of this work, then the matrix $\mathbf{W}\left(\omega_{l}\right)$ is decomposed as, $\mathbf{W}\left(\omega_{l}\right)^{T}=$ $\left[\begin{array}{ll}\mathbf{W}_{1}\left(\omega_{l}\right) & \mathbf{W}_{2}\left(\omega_{l}\right)\end{array}\right]$ so that for the user $j$

$$
\begin{aligned}
& \mathbf{W}_{1}\left(\omega_{l}\right)=\mathbf{M}^{-\mathbf{1}}\left(\omega_{\mathbf{l}}\right) \overline{\mathbf{H}}_{\mathbf{1}}\left(\omega_{\mathbf{l}}\right) \\
& \mathbf{W}_{2}\left(\omega_{l}\right)=\mathbf{M}^{-\mathbf{1}}\left(\omega_{\mathbf{l}}\right) \overline{\mathbf{H}}_{\mathbf{2}}\left(\omega_{\mathbf{l}}\right)
\end{aligned}
$$

and likewise for the $j^{\prime}$ user

$$
\begin{aligned}
& \mathbf{W}_{1}\left(\omega_{l}\right)=\mathbf{M}^{-\mathbf{1}}\left(\omega_{\mathbf{l}}\right) \overline{\mathbf{H}}_{\mathbf{3}}\left(\omega_{\mathbf{l}}\right) \\
& \mathbf{W}_{2}\left(\omega_{l}\right)=\mathbf{M}^{-\mathbf{1}}\left(\omega_{\mathbf{l}}\right) \overline{\mathbf{H}}_{\mathbf{4}}\left(\omega_{\mathbf{l}}\right)
\end{aligned}
$$

These weight vectors are used to estimate $\mathbf{S}^{j}\left(\omega_{l}\right)$ and $\mathbf{S}^{j^{\prime}}\left(\omega_{l}\right) \quad \forall \omega_{l}$, and then an IDFT is applied to the entire block before advanced error correction with a de-interleaver and Viterbi decoder followed by slicer to yield the estimation of the $j$ th user input. This concludes the first step.

\subsection{Co-Channel interference cancellation}

In the second step, we re-estimate the transmitted symbols based on a modified received signal obtained by canceling the co-channel interferences. With the assumption that the signals from the first step have been decoded correctly, a perfect interference cancellation may be achieved by using the second step. In the case of two users, the estimated signals from the first step, for both users, are converted to the frequency domain with DFTs and then equations (5 and (6) are modified as

$$
\begin{aligned}
\dot{\mathbf{R}}\left(1, \omega_{l}\right) & =\mathbf{R}\left(1, \omega_{l}\right)-\mathbf{G}_{\mathbf{1}}\left(\omega_{\mathbf{l}}\right) \hat{\mathbf{S}}^{\mathbf{j}^{\prime}}\left(\omega_{\mathbf{l}}\right) \\
& \approx \mathbf{H}_{\mathbf{1}} \mathbf{S}^{\mathbf{j}}\left(\omega_{\mathbf{l}}\right)+\mathbf{V}_{\mathbf{1}}\left(\omega_{\mathbf{l}}\right) \\
\dot{\mathbf{R}}\left(2, \omega_{l}\right) & =\mathbf{R}\left(2, \omega_{l}\right)-\mathbf{H}_{\mathbf{2}}\left(\omega_{\mathbf{l}}\right) \hat{\mathbf{S}}^{\mathbf{j}}\left(\omega_{\mathbf{l}}\right) \\
& \approx \mathbf{G}_{\mathbf{2}} \mathbf{S}^{\mathbf{j}^{\prime}}\left(\omega_{\mathbf{l}}\right)+\mathbf{V}_{\mathbf{2}}\left(\omega_{\mathbf{l}}\right)
\end{aligned}
$$

The procedure described in the first step is then repeated to re-estimate the transmitted symbols.

Due to its high complexity for certain applications, the proposed scheme is limited in this work for two users. Extension to a larger number of users is necessary for practical applications. Other iterative techniques such as turbo decoding could also be applied. These improvements are subjects of future work.

\section{SIMULATION RESULTS}

In order to illustrate the basic concept of the proposed two-step receiver structure, we run MATLAB-based simulations for a two user space-time block coded MIMO-SCFDE system equipped with two transmit antennas for each

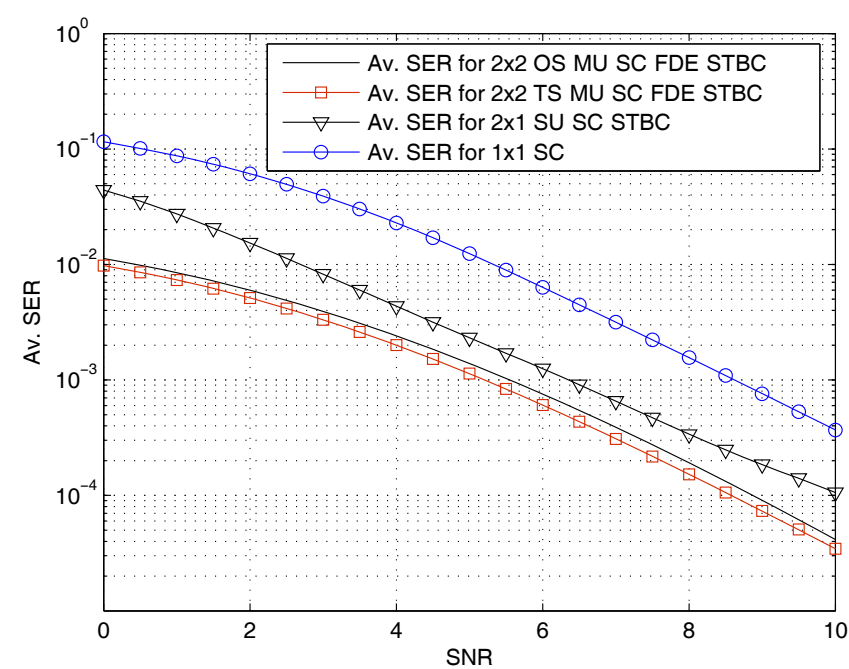

Figure 3. Average symbol error rate simulation results for quasi-static fading channel.

user and two receive antennas. Each block of the user data stream contains 64 BPSK symbols. TR-STBC is applied on each of the two inputs after passing each one through a standard half rate and constraint of three convolutional encoder. Then, a random bit interleaving is applied to correct the carrier in deep fades. An additional length eight cyclic prefix symbol is used as a guard interval after each data block. The data transmission is implemented over two types of channel namely: MIMO frequency selective channels with quasi-static two tap FIR filters, the coefficients are chosen randomly from a normalized complex Gaussian distribution for each block, and MIMO frequency selective channels with slowly time varying fading generated by the modified Jakes fading model [10]. At the receiving end the two-step receiver discussed earlier is employed for both modelled channels, and perfect channel knowledge is assumed.

Firstly, Fig 3 shows a comparison of system performance between four cases namely: basic SISO-SC, single user two transmit one receive STBC-SC-MISO, the output of the first step of our proposed receiver (OS-MU-SC-FDESTBC), and the output of its second step (TS-MU-SC-FDESTBC). The two-step interference cancellation scheme over the range of SNR in the figure shows good performance in terms of the average symbol error rate curve generally better than the other cases, as a result, importantly the ISI and CCI have been canceled effectively by the two-step processing. In this paper, we use the TR-STBC scheme as in [3] and the MMSE scheme in [7], hence the diversity is $n_{t} \times\left(m_{r}-K+1\right)$, independent of that due to the memory of the channel [5]. 


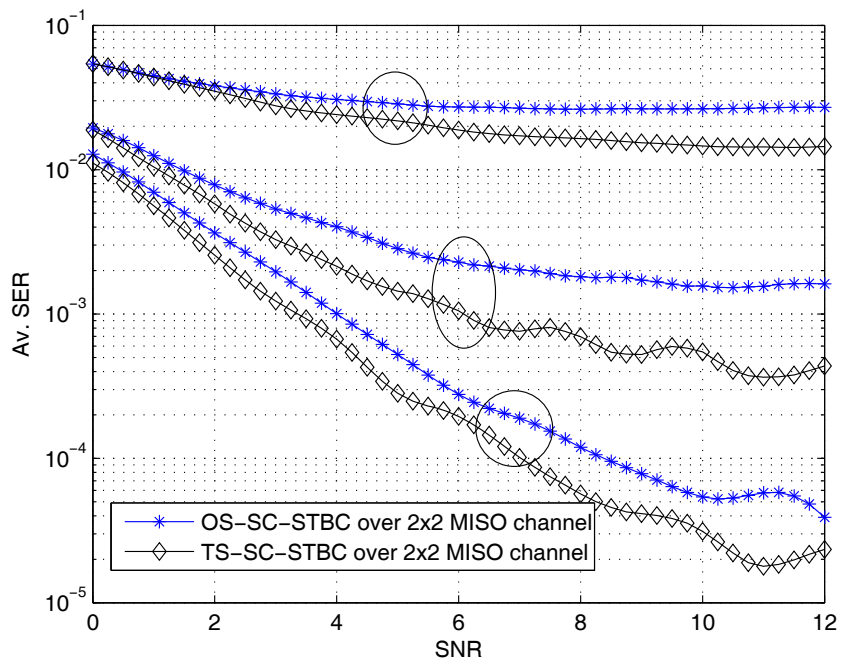

Figure 4. Average symbol error rate simulation results for Jakes' model fading channel with 10,20 , and $40 \mathrm{Km} / \mathrm{h}$ velocities.

Secondly, Fig 4 shows that the performance degrades at higher SNR values with increasing maximum Doppler frequency. Recalling that the channel is assumed to be quasistatic over two consecutive time slots, this assumption is violated with the Jakes' model so the model fails to keep the necessary orthogonality between the antennas and the carrier which causes the error floor. However, ISI and CCI are still suppressed effectively by the coded two step scheme. Importantly, the two step cancelation technique degradation is better than the one step technique.

\section{Conclusion}

In this paper, we have addressed the design of a two-step interference cancellation transceiver to cancel co-channel interference (CCI) in a multiuser MIMO broadband wireless communication system within a quasi-static channel environment. In this approach, the transmitter uses timereversed-space-time block coding modulation, which exploits serial concatenation and single carrier transmission which crucially overcomes the PAPR problem in OFDM systems whilst retaining the advantage in terms of mitigating frequency selective channels. The receiver is based on a two-step interference cancellation algorithm. The simulation results indicate that the proposed scheme could obtain performance improvement in typical wireless communication environments with realizable computational complexity.

By concatenating the transmitter with the half rate outer channel code, the proposed technique obtained significant performance gain over the normal technique.

\section{References}

[1] N. Al-Dhahir, "Single-carrier frequency-domain equalization for space-time block-coded transmissions over frequency-selective fading channels," Communications Letters, IEEE, vol. 5, no. 7, pp. 304-306, 2001.

[2] E. Lindskog and A. Paulraj, "A transmit diversity scheme for channels with intersymbol interference," IEEE International Conference on Communications, 2000. ICC 2000.

[3] Z. Shengli and G. B. Giannakis, "Single-carrier space-time block-coded transmissions over frequencyselective fading channels," IEEE Transactions on Information Theory, vol. 49, no. 1, pp. 164-179, 2003.

[4] A. Paulraj, R. Nabar, and D. Gore, Introduction to space-time wireless communications. Cambridge University Press, 2003.

[5] G. Giannakis, Z. Liu, X. Ma, and S. Zhou, Space Time Coding for Broadband Wireless Communications. Hoboken, New Jersey: John Wiley, 2007.

[6] G. Li and H. Yih-Fang, "A multi-user SC-FDE-MIMO system for frequency-selective channels," IEEE 39th Asilomar Conf, pp. 1593-1597, 2005.

[7] A. Naguib, N. Seshadri, and A. Calderbank, "Applications of space-time block codes and interference suppressionfor high capacity and high data rate wireless systems," IEEE 32nd Asilomar Conf, vol. 2, pp. 18031810, 1998.

[8] Z. Li, M. Sellathurai, and J. A. Chambers, "A joint coded two-step multiuser detection scheme for MIMO-OFDM system," IEEE ICASSP07, vol. 3, pp. III-85-III-88, 2007.

[9] S. Haykin, Communication Systems. Wiley, 1994.

[10] P. Dent, G. E. Bottomley, and T. Croft, "Jakes' fading model revisited," Electronics Letters, vol. 29, no. 13, pp. 1162-1163, 1993. 